\title{
BMJ Open Speaking out about physical harms from tobacco use: response to graphic warning labels among American Indian/Alaska Native communities
}

\author{
David A Patterson Silver Wolf (Adelv unegv Waya), ${ }^{1}$ Molly Tovar, ${ }^{2}$ \\ Kellie Thompson, ${ }^{1}$ Jamie Ishcomer, ${ }^{2}$ Matthew W Kreuter, ${ }^{3}$ Charlene Caburnay, ${ }^{3}$ \\ Sonia Boyum ${ }^{3}$
}

To cite: Patterson Silver Wolf (Adelv unegv Waya) DA, Tovar M, Thompson K, et al. Speaking out about physical harms from tobacco use: response to graphic warning labels among American Indian/Alaska Native communities. BMJ Open 2016;6:e008777.

doi:10.1136/bmjopen-2015008777

- Prepublication history for this paper is available online. To view these files please visit the journal online (http://dx.doi.org/10.1136/ bmjopen-2015-008777).

Received 15 May 2015 Revised 3 October 2015 Accepted 16 October 2015

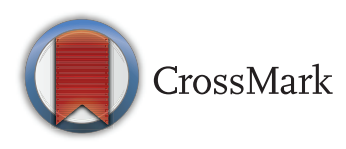

${ }^{1}$ Brown School of Social Work, Washington University in St. Louis, St. Louis, Missouri, USA

${ }^{2}$ Kathryn M Buder Center for American Indian Studies, Washington University in St. Louis, St. Louis, Missouri, USA

${ }^{3}$ Health Communication Research Laboratory, Washington University in St. Louis, St. Louis, Missouri, USA

Correspondence to Dr David A Patterson-Silver Wolf (AdelvunegvWaya); dpatterson22@wustl.edu

\section{ABSTRACT}

Objective: This study is the first to explore the impact of graphic cigarette labels with physical harm images on members of American Indian/Alaska Native (AI/AN) communities. The aim of this article is to investigate how Al/AN respond to particular graphic warning labels.

Methods: The parent study recruited smokers, at-risk smokers and non-smokers from three different age groups (youths aged 13-17 years, young adults aged 18-24 years and adults aged 25+ years) and five population subgroups with high smoking prevalence or smoking risk. Using nine graphic labels, this study collected participant data in the field via an iPadadministered survey and card sorting of graphic warning labels. This paper reports on findings for Al/AN participants.

Results: After viewing graphic warning labels, participants rated their likelihood of talking about smoking risks to friends, parents and siblings higher than their likelihood of talking to teachers and doctors. Further, this study found that certain labels (eg, the label of the toddler in the smoke cloud) made them think about their friends and family who smoke.

Conclusions: Given the influence of community social networks on health beliefs and attitudes, health communication using graphic warning labels could effect change in the smoking habits of Al/AN community members. Study findings suggest that graphic labels could serve as stimuli for conversations about the risks of smoking among Al/AN community members, and could be an important element of a peer-to-peer smoking cessation effort.

\section{BACKGROUND}

Tobacco-related health problems in the general USA population continue to be a major concern. In 2011, an estimated 43.8 million (19\%) US adults were current cigarette smokers. ${ }^{1}$ One in five deaths, approximately 443000 each year in the US, can be

\section{Strengths and limitations of this study}

- This study had a total sample size of 220 American Indian and Alaska Natives with varying demographics including; age, gender and educational attainment.

- This study catered to the unique needs of the American Indian and Alaska Native population by incorporating special safeguards such as interpreters, talking circles and removal of certain images.

- Reactions were recorded after only one viewing of the images, whereas if these were actual cigarette package labels, participants would have likely viewed the images more often, and reactors or behaviour could have changed due to repeated viewing.

- This study used convenience sampling; therefore these findings cannot be generalised to all American Indian and Alaska Native populations.

attributed to smoking cigarettes. ${ }^{2}$ American Indian/Alaska Native (AI/AN) communities are dangerously impacted by commercial tobacco use. The $36 \%$ prevalence rate of cigarette smoking in $\mathrm{AI} / \mathrm{AN}$ communities is twice the national average (approximately $18 \%$ ). The leading cause of preventable death among AI/AN people is directly related to cigarette smoking. ${ }^{34}$

To address the risks of smoking, text-only Surgeon General's warning labels have been placed on cigarette packs since 1984. Studies investigating reactions to cigarette warning labels suggest that perceived risk increases as warnings are more deeply processed. ${ }^{5}$ Acceptance of warning label messages can be improved if negative emotions (eg, fear, disgust, anger) are salient, as long as the messages are perceived to be credible and not 'too staged'. ${ }^{7}$ Experiencing negative emotions also has been associated with more 
quit attempts and cessation. ${ }^{6}$ Avoidance behaviours such as using a case to hide warning labels or avoiding a particular label at point-of-sale has been reported among adults, although such avoidance was not associated with subsequent quit attempts. ${ }^{6} 8$ The source and content of discussions related to antismoking ads have been shown to affect attitudes and behaviours among adults independent of the ads themselves, ${ }^{9}$ suggesting that antitobacco campaigns are more effective when they stimulate conversation about the ads themselves and about smoking risk.

Seeking to improve the efficacy of warning labels, about 40 countries worldwide have begun to use warning labels with graphic images, although tobacco industry litigation has delayed their use in the USA. ${ }^{10}$ Studies conducted outside the USA have found associations between graphic warning labels and reduction in national smoking rates, and increased quit attempts. ${ }^{5} 1112$ In addition, graphic warning labels appear to be more effective than text-only labels in drawing attention, fostering information processing and message recall, and increasing perceived health risks of smoking. ${ }^{7}{ }^{13-15}$ Increased information processing, in turn, has been associated with greater intention to quit and changes in smoking behaviour. ${ }^{16}$ Graphic labels appear to be effective despite the avoidance behaviours reported by a small number of respondents. ${ }^{7}{ }^{17}$ Some studies have also found that graphic labels may be more effective for particular subpopulations, namely those with low education, smokers and minorities, ${ }^{73-15}$ although they may be less effective for smokers who do not wish to quit. ${ }^{18}$

Studies investigating smoking cessation efforts in AI/ AN communities using graphic images are non-existent, despite disproportionately high smoking rates in these communities. ${ }^{19}$ The aim of this article is to investigate which graphic warning labels elicit the most response in $\mathrm{AI} / \mathrm{AN}$ communities across the USA, who is impacted most and how this information can be used to reduce the prevalence of harmful tobacco use in $\mathrm{AI} / \mathrm{AN}$ communities. This study is unique in that it is the first large national study on the impact of cigarette warning labels that includes targeted recruitment of AIs/ANs where they work and live.

\section{METHODS}

\section{Sample and recruitment}

This paper reports on findings for AIs/ANs who participated in a larger study about graphic warning labels (June 2012-March 2013). For the larger study, targeted recruitment through community partners in 14 states was used to enrol a diverse convenience sample of participants from five population subgroups with high rates of smoking and/or smoking-related morbidity and mortality: low-income and rural Americans, blacks, AI/AN, US military personnel and blue-collar workers. ${ }^{20-22}$ Recruitment venues for $\mathrm{AI} / \mathrm{AN}$ in southwestern and eastern states included powwows, reservations, health fairs and tribal organisation events. Owing to the sample being a special population needing additional protections, special safeguards were in place, if needed, such as interpreters, talking circles and removal of certain images. The total sample size was 1571 , of which 220 were AI/AN. All study procedures were approved by the Institutional Review Board at Washington University in St. Louis.

\section{Study design}

This study collected participant data in the field via a coordinated series of face-to-face research activities. The sequence of these activities involved completing a baseline assessment, a survey regarding graphic warning labels, a card sort exercise, an in-depth interview and a randomised follow-up study. All participants in the study activities received remuneration for their time. Respondents received a $\$ 25$ gift card for completing the baseline assessment, survey and card sort activity, and a $\$ 50$ gift card for the in-depth interview. Respondents in the randomised follow-up study received renumeration based on the number of follow-up assessments completed. ${ }^{23}$

The data reported in this paper were collected from the baseline assessment and survey. These two activities were completed by participants on iPads that were programmed to guide participants through a series of questions. Project staff distributed iPads to participants and gave directions on how to use the iPad interface. At some recruitment locations, iPads were distributed and directions given-one participant at a time. At other locations, iPads were distributed to participants in a classroom setting and directions were delivered to all participants at the same time. Interpreters were utilised at the participant's request if English was not the primary language. Approximately $26 \%$ of the recruitment locations utilised group administration of surveys, and $68.5 \%$ used individual administration, whereas $5.5 \%$ used both methods. All project staff received the same training on providing instruction on iPad use. Beyond these very basic instructions on navigating the iPad interface, participants were guided by directions delivered via the iPad itself. For this reason, no validity checks were performed.

\section{Baseline assessment and survey}

Participants completed a brief iPad-administered baseline assessment that requested information on participant characteristics of interest, including demographics (eg, age, race/ethnicity), smoking-related variables (eg, smoking status of self, peers and family, amount and duration of smoking, nicotine dependence) and literacy.

Participants then completed a 30 min survey on iPads. The survey consisted of a presentation on the iPad interface of nine graphic warning labels with images of physical harm caused by smoking (figure 1). Participants were shown a single warning label at a time, in random order, accompanied by a question about the label. 

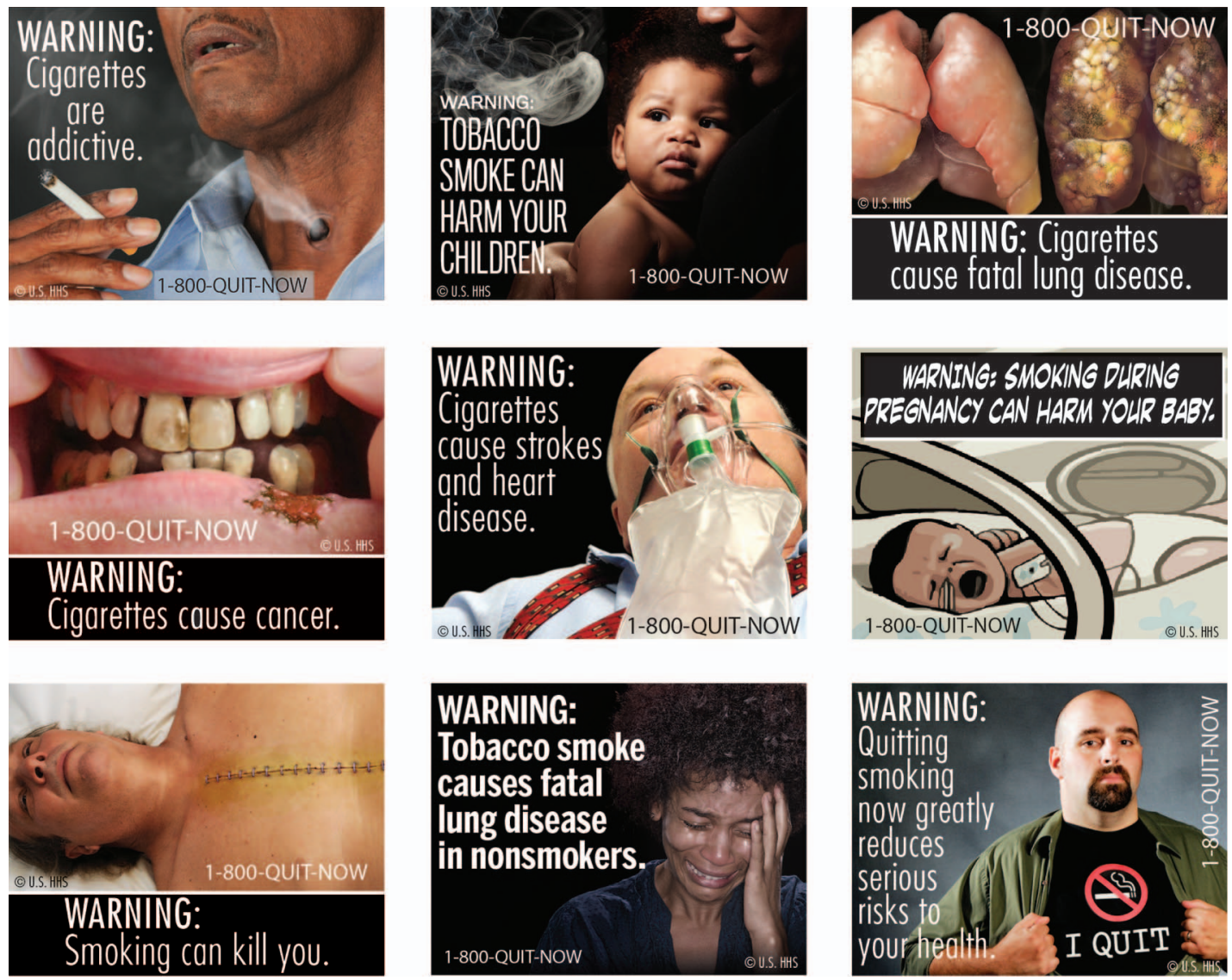

Figure 1 Warning labels.

Participants responded to the question by pressing the response on the iPad. Because it is a taboo to view or handle certain parts of a body in many $\mathrm{AI} / \mathrm{AN}$ cultures, ${ }^{24} \mathrm{AI} / \mathrm{AN}$ participants were asked a screening question at the beginning of the survey: 'With respect to traditional tribal values and beliefs, we want to inform you of images and words which may go against your tribal practices. Some of the images include a cadaver, lungs and heart. Are you able and willing to look at these pictures?'. Participants who replied no to this statement were not shown the diseased lungs and cadaver images. Another cultural consideration regarding smoking in AI/AN communities is traditional and ceremonial uses of tobacco. Our study did not explore this complex relationship because the focus was on commercial marketing of cigarettes and other tobacco products.

\section{Measures}

Participant characteristics of interest in the study included demographics (eg, age, gender) and smoking-related variables (eg, smoking status of self, peers and family, amount and duration of smoking, nicotine dependence). Categories of smoking behaviour were determined separately for youth, young adults and adults. Youth were assigned to one of three smoking categories: smokers (smoked cigarettes in the past 30 days), non-smokers (never smoked cigarettes in the past 30 days or never tried a cigarette, even one or two puffs) and at-risk smokers (never tried but had friends or knew adults who smoke). Young adults were assigned as follows: smokers (currently smoking cigarettes every day or some days), non-smokers (have never smoked 100 cigarettes in their entire lives and do not smoke now) and at-risk young adults (have tried cigarettes even one or two puffs and think they will try smoking or are willing to try cigarettes if offered by a friend or will smoke a cigarette next year). Adults were assigned to one of three categories: smokers (currently smoking cigarettes every day or some days), non-smokers (have never smoked 100 cigarettes in their entire lives and do not smoke now) and at-risk adults (have smoked 100 cigarettes in their entire lives and no longer smoke now).

\section{Emotions}

Emotional and affective responses are essential to the effectiveness of health messaging, facilitating greater recall and greater awareness of perceived risk and benefits of smoking. ${ }^{25}$ First, we assessed the degree to which each warning elicits each of five emotions, identified by Ekman $^{26}$ as basic human emotions: disgust, fear, sadness, anger and regret. Two additional basic emotions identified by Ekman, happiness and surprise, were not included in the study measures because of the negative content of the images. Regret was also assessed because other research has identified this emotion as a 
common emotion among smokers. ${ }^{27}{ }^{28}$ For each label, we asked participants to rate their emotional response on a seven-point scale ( $1=$ not at all to $7=$ completely) for five different emotions with the question 'While looking at the [label \#], I felt: disgusted or grossed out, worried, sad, regretful, angry'. Those who rated an emotion as greater than one were then asked a series of follow-up questions. For example, participants who indicated some anger (anger rating $>1$ ), were asked 'I feel angry at: people who smoke; people who smoke around me; companies that make cigarettes' (yes/no). Smokers who indicated some anger were also asked 'I feel angry at: myself for being a smoker; the warning labels and its sponsors' (yes/no).

\section{Communication}

Graphic warning labels on cigarettes might elicit social reactions that contribute to reduced smoking. For example, a smoker's spouse or partner, friend, co-worker or child might express concern overseeing the warnings. Thus, we assessed whether exposure to the warning labels stimulated the intent to communicate about the labels. Communication actions would include talking about the warnings with others (eg, coworkers, peers, children, parents, friends) and would apply equally to smokers and non-smokers. For each label, participants were asked, 'How likely would you be to talk about this label with (a) friends who smoke, (b) parents (children), (c) siblings, (d) teachers (coworkers), (e) doctors?'. Response options on a 7-point Likert scale ranged from 1 (not at all likely) to 7 (extremely likely). Only participants with friends or family who smoked were asked question (a). The whole sample was asked questions (b)-(e). Participants were also asked, 'If you and your friends were looking at these warning labels together, which ones do you think you would talk about the most? You can choose up to three'. For each label, we recorded a dichotomous variable (yes/no) to indicate whether or not the label was chosen.

\section{Thinking about friends and family who smoke}

Different warning labels may vary in their ability to stimulate self-referential thinking in different subgroups of the target population. For example, men and women viewing the same warning label may rate its personal relevance differently. We assessed the personal relevance of the labels for those who had family and friends who smoke. For each label, participants were asked, 'This label makes me think about my family members who smoke'. Participants indicated their response by adjusting a slider between 0 (not at all) and 100 (a lot). The perceived harm of smoking was assessed by asking 'Looking at the label makes me feel like smoking could hurt the health of my close friends or family members'. Response options ranged on a 7-point Likert scale from 1 (not at all) to 7 (completely). In addition, participants with friends who smoked were asked, 'Do any of these warning labels make you think about your friends?'. Participants could choose up to three labels. Those with family who smoked were asked, 'Do any of these warning labels make you think about people in your family?'. Again, participants could choose up to three labels. For each label, we recorded a dichotomous variable (yes/no) to indicate whether or not the label was chosen.

\section{Data analysis}

Descriptive statistics were calculated for all measures, including means and SDs for continuous and Likert scale variables and percentages for dichotomous variables. Statistical analyses were conducted using SAS V.9.4. Any missing data was excluded by list-wise deletion. A three-way analysis of variance was conducted to assess emotional response by sex, age group and smoking status. Differences in least-squares means were compared and adjusted for multiple comparisons by the Tukey Cramer method. Logistic regression analyses were conducted for each label using sex, age group and smoking status as covariates. ORs and 95\% CIs were estimated.

\section{RESULTS}

Table 1 summarises the demographic characteristics of the $\mathrm{AI} / \mathrm{AN}$ sample. Of the $\mathrm{AI} / \mathrm{AN}$ in the study, 25\% were non-smokers, whereas $47.3 \%$ were at-risk smokers and $27.7 \%$ were smokers; $75.9 \%$ had close friends who smoked; $63 \%$ of youths had parents or caretakers who smoked; and $73.7 \%$ of adults had close family members who smoked. The sample included $37.6 \%$ males and $62.4 \%$ females; $27 \%$ were aged $13-17$ years, $28.2 \%$ were aged 18-24 years and adults aged 25 years and older composed $44.5 \%$ of the study participants. Ninety per cent of the AI/AN participants viewed every label, whereas $10 \%$ viewed only seven of the nine.

\begin{tabular}{lrc} 
Table $1 \quad$ Demographics $(\mathrm{n}=220)$ & & \\
\hline & $\mathbf{n}$ & Per cent \\
\hline Age (years) & 60 & 27.3 \\
$13-17$ & 62 & 28.2 \\
$18-24$ & 98 & 44.5 \\
$25+$ & & \\
Gender & 82 & 37.6 \\
$\quad$ Male & 136 & 62.4 \\
$\quad$ Female & & \\
Smoking status & 55 & 25.0 \\
$\quad$ Non-smokers & 104 & 47.3 \\
At-risk smokers & 61 & 27.7 \\
$\quad$ Smokers & 76 & 38.8 \\
Education & 53 & 27.0 \\
$\quad$ Less than high school & 16 & 8.2 \\
$\quad$ High school graduate or GED & 26 & 13.3 \\
$\quad$ Vocational training & 25 & 12.8 \\
$\quad$ Four-year college degree & & \\
$\quad$ Some graduate studies or more & 25 \\
GED, General Education Development. & & \\
$\quad$ & &
\end{tabular}




\section{Emotions}

Overall, there were few significant differences regarding emotional response rates to the various labels, but the significant differences that were found are worthy of note. The labels depicting the child in the smoke cloud and the baby in the incubator had the highest mean anger, sadness and worry among all AI/AN participants. However, there was a significant difference in anger ratings for the baby in the incubator image among smokers and those at risk, with smokers rating their anger lower than those at risk. The labels depicting a diseased lung and cadaver had lower emotion ratings overall than the child in the smoke cloud and the baby in the incubator. There was a significant difference, though, in anger rating between women and men for the lung and cadaver labels, with $\mathrm{AI} / \mathrm{AN}$ women rating their anger significantly higher than men for these labels (figure 2).

Of those who indicated some anger after viewing the label depicting the child in the smoke cloud, 52.8\% responded 'yes' to the option 'I am angry at people who smoke', while $48.3 \%$ responded 'yes' to the option 'I am angry at people who smoke around me', and $57.4 \%$ responded 'yes' to the option 'I am angry at companies that make cigarettes'. For those who responded with some anger after viewing the label with the baby in the

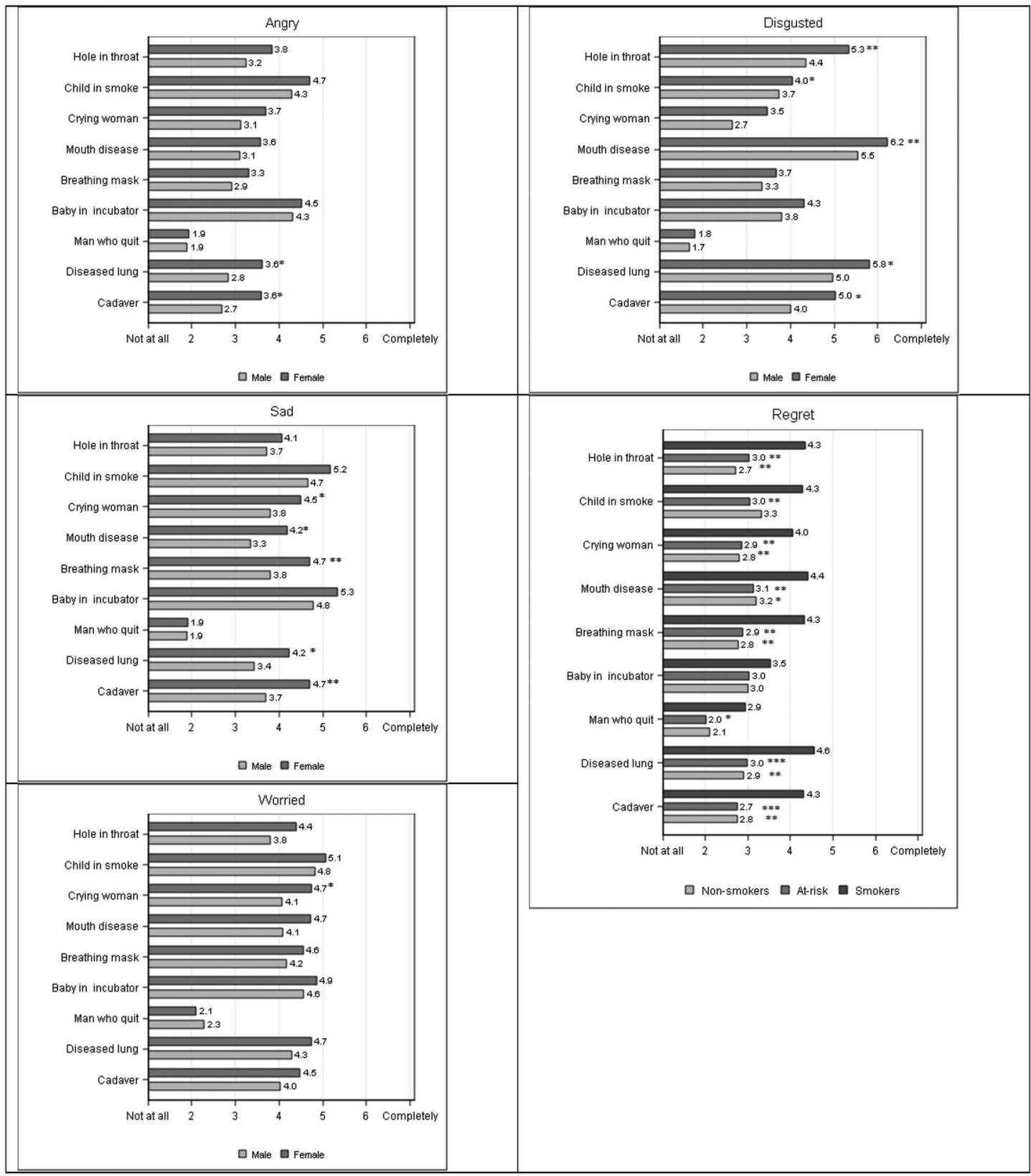

Figure 2 Adjusted means for emotions by sex or smoking status. Models included sex, age group and smoking status. Significant differences between means for males versus females or for smokers versus at-risk smokers or non-smokers. ${ }^{*} p<0.05$; ${ }^{* *} \mathrm{p}<0.01 ;{ }^{* * *} \mathrm{p}<0.001$. 
incubator, $57.8 \%$ responded 'yes' to the option 'I am angry at people who smoke', $54.3 \%$ responded 'yes' to the option 'I am angry at people who smoke around me', and $41.6 \%$ responded 'yes' to the option 'I am angry at companies that make cigarettes'. Over $60 \%$ of those expressing anger for the labels showing the hole in the throat, the diseased mouth, the breathing mask and the cadaver indicated that they were angry at companies that make cigarettes.

Disgust was highest after viewing the label depicting the diseased mouth. Other labels eliciting high disgust were the diseased lung and the hole in the throat. Women rated their disgust significantly higher than men for the hole in the throat, the crying woman, the diseased mouth, the diseased lung and the cadaver labels. Those aged 13-17 years showed significantly higher disgust than adults after viewing the labels showing hole in the throat and the diseased mouth. There were no significant differences in disgust by smoking status.

Some images also elicited sadness, worry and regret. The images of the crying woman, the diseased mouth, the breathing mask, the diseased lung and the cadaver elicited higher sadness from AI/AN women than men. Youths reported higher sadness after viewing the diseased mouth than did young adults. There were no significant differences in sadness between smoking status groups for any of the labels. Regarding worry, women reported significantly higher worry than men after viewing the label with the crying woman. Non-smokers reported lower worry than smokers after viewing the label depicting the diseased mouth. There were no significant differences in worry between age groups. Regret was higher for smokers than for other smoking status groups, whereas levels of regret were similar for men and women. Smokers reported higher regret than at-risk participants after viewing the child in the smoke cloud and the man who quit. Smokers also had higher regret than at-risk smokers and non-smokers after viewing the hole in the throat, the crying woman, the diseased mouth, the breathing mask, the diseased lung and the cadaver. Adults reported significantly higher regret compared to youths after viewing the diseased lung.

\section{Talking about the labels}

There was not much variation between labels regarding which label would most likely be talked about to friends who smoke, siblings, teachers or doctors (see table 2). Participants rated their likelihood to talk to friends who smoke, parents (children) and siblings higher than talking to teachers or doctors. The labels most often chosen as labels that participants would talk about most with friends were the diseased mouth $(54.5 \%)$, the diseased lung $(46.0 \%)$ and the hole in the throat $(45.5 \%)$. The labels chosen the least often were the crying woman $(14.6 \%)$, the breathing mask $(15.5 \%)$ and the man who quit $(14.1 \%)$. The crying woman was more likely to be chosen by youths than by adults $(\mathrm{OR}=2.91$; CI 1.07 to 7.95$)$. The baby in the incubator was more likely to be chosen by young adults compared to adults (OR=3.54; CI 1.60 to 7.80). At-risk smokers were less likely to choose the label depicting the diseased lung compared to non-smokers $(\mathrm{OR}=0.40$; CI 0.18 to 0.86 ).

\section{Thinking about friends and family who smoke}

The labels depicting the child in the smoke cloud, the diseased lung and the cadaver had the highest rating for 'making me think about my family members who smoke' (see table 2). These were also the labels with the highest scores for the question 'looking at the label makes me feel like smoking could hurt the health of my close friends or family members'. The two labels most often chosen that made participants think about friends who smoke were the child in the smoke cloud (45.6) and the label depicting mouth disease $(47.1 \%)$. The two labels most often chosen for thinking about family were the child in the smoke cloud $(45.7 \%)$ and the diseased lung $(43.5 \%)$.

\section{DISCUSSION}

This study demonstrates the impact of labels with physical harm images on members of the AI/AN community. The results demonstrate that graphic warning labels elicited emotional responses in participants, including anger, sadness, worry and regret. There were few significant differences in reaction to particular graphic images, although participants reacted with stronger anger, sadness and worry to graphic labels depicting children. Of those reporting an angry response, roughly equal number of respondents reported that they were angry at people who smoke, angry at people who smoke around them and angry at companies that make cigarettes. Although the difference was not statistically significant, particular images appeared to elicit more anger at companies that make cigarettes than others. There were some statistically significant differences in emotional response to labels by gender, age and smoking status, suggesting that some images may be more effective than others for certain subpopulations. . Studies investigating reactions to cigarette warning labels suggest that labels are more effective at increasing perception of risk if they elicit negative emotions such as disgust, anger and regret. ${ }^{6}{ }^{7}$ Experiencing negative emotions also has been associated with more quit attempts and cessation. ${ }^{6}$

In addition to measuring the emotional response, this study also examined the likelihood of graphic warning labels to stimulate discussion among participants and to make participants think about friends and family members who smoke. There were few differences in labels by likelihood of stimulating conversation, although participants rated the labels depicting physical harms (diseased mouth, disease lung and hole in throat) as those they were most likely to discuss with 
Table 2 Friends and family who smoke

\begin{tabular}{|c|c|c|c|c|c|c|c|c|c|}
\hline & \multicolumn{9}{|l|}{ Mean (SD) } \\
\hline & $\begin{array}{l}\text { Hole in } \\
\text { throat }\end{array}$ & $\begin{array}{l}\text { Toddler in } \\
\text { smoke } \\
\text { cloud }\end{array}$ & $\begin{array}{l}\text { Crying } \\
\text { woman }\end{array}$ & $\begin{array}{l}\text { Mouth } \\
\text { disease }\end{array}$ & $\begin{array}{l}\text { Breathing } \\
\text { mask }\end{array}$ & $\begin{array}{l}\text { Baby in } \\
\text { incubator }\end{array}$ & $\begin{array}{l}\text { Man who } \\
\text { quit }\end{array}$ & $\begin{array}{l}\text { Diseased } \\
\text { lung }\end{array}$ & Cadaver \\
\hline \multicolumn{10}{|c|}{ How likely would you be to talk about this label with ( $1=$ not at all likely, $7=$ extremely likely) } \\
\hline A. Friends who smoke? & $4.6(2.17)$ & $4.6(2.26)$ & $4.1(2.31)$ & $4.6(2.15)$ & $4.2(2.24)$ & $4.4(2.27)$ & $4.1(2.31)$ & $4.6(2.21)$ & $4.5(2.13)$ \\
\hline B. Parents (children) & $4.7(2.26)$ & $4.9(2.20)$ & $4.4(2.31)$ & $4.6(2.24)$ & $4.5(2.3)$ & $4.6(2.32)$ & $4.2(2.33)$ & $4.6(2.28)$ & $4.5(2.28)$ \\
\hline C. Siblings? & $4.4(2.32)$ & $4.6(2.34)$ & $4.3(2.34)$ & $4.6(2.35)$ & $4.3(2.34)$ & $4.5(2.36)$ & $4.3(2.36)$ & $4.4(2.37)$ & $4.5(2.31)$ \\
\hline D. Teachers (coworkers)? & $3.8(2.37)$ & $3.9(2.40)$ & $3.6(2.30)$ & $3.8(2.36)$ & $3.6(2.27)$ & 3.7 (2.39) & 3.7 (2.30) & 3.7 (2.31) & $3.6(2.27)$ \\
\hline E. Doctors? & $4.0(2.44)$ & $4.0(2.49)$ & 3.7 (2.44) & $4.0(2.38)$ & 3.8 (2.38) & $3.9(2.50)$ & 3.7 (2.42) & $3.9(2.41)$ & 3.8 (2.38) \\
\hline $\begin{array}{l}\text { This label makes me think about my } \\
\text { family members who smoke }(1-100)\end{array}$ & $65.3(33.95)$ & $68.3(32.98)$ & $63.5(34.05)$ & $66.8(33.56)$ & $64.7(33.15)$ & $60.9(36.74)$ & $55.8(35.20)$ & $69.5(32.36)$ & $69.9(32.47)$ \\
\hline $\begin{array}{l}\text { Looking at the label makes me feel like } \\
\text { smoking could hurt the health of my } \\
\text { close friends or family members } \\
\text { (1=not at all, } 7=\text { completely) }\end{array}$ & $5.6(1.83)$ & $5.9(1.61)$ & $5.6(1.72)$ & $5.6(1.71)$ & $5.6(1.76)$ & $5.6(1.78)$ & $4.4(2.27)$ & $5.9(1.66)$ & $5.77(1.6)$ \\
\hline $\begin{array}{l}\text { If you were looking at these warning } \\
\text { labels together, which ones do you } \\
\text { think you would talk about the most? } \\
\text { (choose 3) }\end{array}$ & $45.5 \%$ & $40.9 \%$ & $14.6 \%$ & $54.5 \%$ & $15.5 \%$ & $33.3 \%$ & $14.1 \%$ & $46.0 \%$ & $28.6 \%$ \\
\hline $\begin{array}{l}\text { Do any of these warning labels make } \\
\text { you think about your friends? } \\
\text { (choose 3) }\end{array}$ & $36.8 \%$ & $45.6 \%$ & $27.9 \%$ & $47.1 \%$ & $16.9 \%$ & $36.8 \%$ & $18.4 \%$ & $39.7 \%$ & $22.8 \%$ \\
\hline $\begin{array}{l}\text { Do any of these warning labels make } \\
\text { you think about your family? } \\
\text { (choose 3) }\end{array}$ & $26.1 \%$ & $45.7 \%$ & $31.2 \%$ & $31.9 \%$ & $30.4 \%$ & $26.8 \%$ & $18.8 \%$ & $43.5 \%$ & $36.2 \%$ \\
\hline
\end{tabular}


riends and family. Interestingly, participants rated their likelihood of talking to friends who smoke, parents (children) and siblings higher than talking to teachers or doctors, suggesting that graphic warning labels may be most effective at stimulating conversation among peers and family members in AI/AN communities. Participants reported that certain labels (eg, labels depicting physical harms and the label of the toddler in the smoke cloud) made them think about their friends and family who smoke. The source and content of discussions related to antismoking ads have been shown to affect attitudes and behaviours among adults independent of the ads themselves, ${ }^{9}$ suggesting that antitobacco campaigns are more effective when they stimulate conversation about the ads themselves and about smoking risk. In addition, discussing graphic warning labels has been shown to be associated to lower smoking intentions in youth and quit attempts in adults. ${ }^{16}$

Although more research is needed, these findings suggest that graphic warning labels have potential to reduce the prevalence of cigarette smoking in $\mathrm{AI} / \mathrm{AN}$ communities by stimulating emotional response and prompting conversation among peers and family members regarding smoking risks. Given the established literature on $\mathrm{AI} / \mathrm{AN}$ mistrusting medical institutions, ${ }^{29} 30$ it seems particularly noteworthy that participants indicated a higher likelihood of talking to peers and family than 'experts' such as doctors or teachers. Graphic warning labels such as those tested in this study could serve as important stimuli for peer-to-peer and intrafamily health and wellness messages in Native communities. This follows $\mathrm{Fu}$ et $a l \mathrm{~s}^{31}$ recommendations that smoking cessation interventions in Native communities should come from $\mathrm{AI} / \mathrm{AN}$ community members and be based in cultural understandings.

This is the first study known to the authors that examines the impact of graphic warning labels in AI/AN communities. Future research on graphic images is needed to understand any impact on AI/AN communities. Randomly assigning specific communities to receive either graphic warnings showing physical harm images or non-physical harms images could possibly impact community-level prevalence rates over time. Until there are randomised controlled trials within $\mathrm{AI} / \mathrm{AN}$ communities on best approaches to reducing tobacco use, having graphic images on cigarette packs could increase the discussion of the harms of tobacco use in $\mathrm{AI} / \mathrm{AN}$ families.

\section{Limitations}

There are limitations in this study. One limitation is that we only asked participants for reactions after viewing the images once, whereas if these were actual cigarette package labels, they would have been viewed more often, and reactions or behaviour could change because of repeated viewing. Another limitation is the study's use of convenience sampling. Although we obtained a large sample of $\mathrm{AI} / \mathrm{AN}$ representing several hundred federal and state-recognised tribes in the USA, these findings cannot be generalised to all $\mathrm{AI} / \mathrm{AN}$ communities.

\section{CONCLUSION}

Although the mass media approach to smoking cessation is most effective in the general population, ${ }^{32}$ the continued high smoking rates in $\mathrm{AI} / \mathrm{AN}$ communities indicates that a different approach might be needed. A key determinant of health behaviours is the influence of community social networks on health-related beliefs and attitudes. ${ }^{33} 34$ This study's findings suggest that graphic warning labels have the potential to effect change in the smoking habits of AI/AN communities by harnessing the power and influence of $\mathrm{AI} / \mathrm{AN}$ peers and family members.

Acknowledgements The authors would like to thank Pete Coser, Sarah Nelson and Choi Lai for their assistance with data collection and analysis. They also thank all the American Indian and Alaska Native tribal nations and communities who allowed them to work within their areas.

Contributors DAP-SW and MT wrote the paper with important revisions provided from all coauthors. KT and JIr helped with literature review. MWK and CC designed the study and supervised the fieldwork. SB conducted the statistical analysis. DAP-SW gave final approval of the manuscript.

Funding This work was supported by the National Cancer Institute and the Family Smoking Prevention and Tobacco Control Act grant number 3P50-CA05815. The content is solely the responsibility of the authors and does not necessarily represent the official views of the National Institutes of Health (NIH) or the Food and Drug Administration. Additionally, prior to issuing a press release concerning the outcome of this research, please notify the NIH awarding IC in advance to allow for coordination.

\section{Competing interests None declared.}

Ethics approval Washington University in St. Louis IRB.

Provenance and peer review Not commissioned; externally peer reviewed.

Data sharing statement No additional data are available.

Open Access This is an Open Access article distributed in accordance with the Creative Commons Attribution Non Commercial (CC BY-NC 4.0) license, which permits others to distribute, remix, adapt, build upon this work noncommercially, and license their derivative works on different terms, provided the original work is properly cited and the use is non-commercial. See: http:// creativecommons.org/licenses/by-nc/4.0/

\section{REFERENCES}

1. Schiller JS, Lucas JW, Peregoy JA. Summary health statistics for U.S. adults: national health interview survey, 2011. Vital Health Stat 2012;10:1-207.

2. U.S. Department of Health and Human Services. How tobacco smoke causes disease: The biology and behavioral basis for smoking-attributable disease. A report of the surgeon general. Atlanta, GA: National Center for Chronic Disease Prevention and Health Promotion, Office on Smoking and Health, 2010. http://www. surgeongeneral.gov/library/reports/tobaccosmoke/index.html (accessed 20 Oct 2013).

3. Centers for Disease Control and Prevention (CDC). Mortality among American Indians and Alaska Natives-United States, 1994-1998. MMWR Morb Mortal Wkly Rep 2003;52:704-7.

4. U.S. Department of Health and Human Services. The tobacco use and dependence clinical practice guideline panel staff and consortium representatives. A clinical practice guideline for treating tobacco use and dependence. J Am Med Assoc 2000;283:3244-54. 
5. Fathelrahman Al, Li L, Borland R, et al. Stronger pack warnings predict quitting more than weaker ones: finding from the ITC Malaysia and Thailand surveys. Tob Induc Dis 2013;11:20-7.

6. Hammond D, MacDonald PW, Fong GT, et al. Do smokers know how to quit? Knowledge and perceived effectiveness of cessation assistance as predictors of cessation behaviour. Addiction 2004:99:1042-8.

7. Hammond D. Health warning messages on tobacco products: a review. Tob Control 2011;20:327-37.

8. Borland $\mathrm{R}$, Yong $\mathrm{HH}$, Wilson $\mathrm{N}$, et al. How reactions to cigarette packet health warnings influence quitting: findings from the ITC four-country survey. Addiction 2009;104:669-75.

9. Samu S, Bhatnagar N. The efficacy of anti-smoking advertisements: the role of source, message, and individual characteristics. Int $J$ Nonprofit Volunt Sect Mark 2008;13:237-50.

10. Canadian Cancer Society. Cigarette package warning labels: International status report. Ottawa: Canadian Cancer Society, 2010:10.

11. Huang J, Chaloupka FJ, Fong GT. Cigarette graphic warning labels and smoking prevalance in Canada: a critical examination and reformulation of the FDA regulatory impact analysis. Tob Control 2014;23(Suppl 1):i7-12.

12. Hammond D, Fong GT, McDonald PW, et al. Showing leads to doing: graphic cigarette warning labels are an effective public health policy. Eur J Public Health 2006;16:223.

13. Cantrell J, Vallone DM, Thrasher JF, et al. Impact of tobacco-related health warning labels across socioeconomic, race and ethnic groups: results from a randomized web-based experiment. PLOS ONE 2013;8:e52206.

14. O'Hegarty M, Pederson LL, Nelson DE, et al. Reactions of young adult smokers to warning labels on cigarette packages. Am J Prev Med 2006;30:467-73.

15. Thrasher JF, Carpenter MJ, Andrews JO, et al. Cigarette warning label policy alternatives and smoking-related health disparities. $A m \mathrm{~J}$ Prev Med 2012;43:590-600.

16. Hammond D, Fong GT, McDonald PW, et al. Impact of the graphic Canadian warning labels on adult smoking behavior. Tob Control 2003;12:391-5.

17. Hammond D, Fong GT, McDonald PW, et al. Graphic Canadian cigarette warning labels and adverse outcomes: evidence from Canadian smokers. Am J Public Health 2004;94:1442-5.

18. Willemsen MC. The new EU cigarette health warnings benefit smokers who want to quit the habit: results from the Dutch Continuous Survey of Smoking Habits. Eur J Public Health 2005;15:389-92.

19. Davis RM, Gilpin EA, Loken B, et al. NCl Tobacco Control Monograph Series: the role of the media in promoting and reducing tobacco use. cancercontrol.cancer.gov/brp/tcrb/monographs/19/ m19 complete.pdf (accessed 2 Nov 2013).

20. Agaku IT, King BA, Dube SR, Centers for Disease Control and Prevention (CDC). Current cigarette smoking among adults-United States, 2005-2012. MMWR Morb Mortal Wkly Rep 2014;63:29-34.

21. DeSantis C, Naishadham D, Jemal A. Cancer statistics for African Americans, 2013. CA Cancer J Clin 2013:63:151-66.

22. Ham DC, Przybeck T, Strickland JR, et al. Occupation and workplace policies predict smoking behaviors: analysis of national data from the current population survey. J Occup Env Med 2011;53:1337-45.

23. McQueen A, Kreuter MW, Boyum S, et al. Reactions to FDA-proposed graphic warning labels affixed to US smokers cigarette packs. Nicotine Tob Res 2015;17:784-95.

24. Arviso L, Cohen Van Pelt E. The scalpel and the silver bear: the first Navajo woman surgeon combines western medicine and traditional healing. New York, USA: Bantam, 2000.

25. Cameron LD, Chan CKY. Designing health communications: harnessing the power of affect, imagery, and self-regulation. Per SocPsyc Comp 2008;2:262-82.

26. Ekman P, Friesen WV, Ellsworth P. Emotion in the human face: guide-lines for research and an integration of findings. New York: Pergamon Press, 1972.

27. Aveyard P, Bauld L. Incentives for promoting smoking cessation: what we still do not know. Cochrane Database Syst Rev 2011;(8): ED000027.

28. Grob G. Major tobacco-related events in the United States, Robert Wood Johnson Foundation (RWJF) Retrospective Series. 2011. http://www.rwjf.org/pr/product.jsp?id=72058.

29. Guadagnolo BA, Cina K, Helbig P, et al. Medical mistrust and less satisfaction with health care among native Americans presenting for cancer treatment. $J$ Health Care Poor Underserved 2009;20:210-26.

30. Fu SS, Burgess D, van Ryn M, et al. Views on smoking cessation methods in ethnic minority communities: A qualitative investigation. Prev Med 2007;44:235-40.

31. Fu SS, Rhodes KL, Robert C, et al. Designing and evaluating culturally specific smoking cessation interventions for American Indian communities. Nicotine Tob Res 2014;16:42-9.

32. National Cancer Institute. The role of the media in promoting and reducing tobacco use. Tobacco Control Monograph No. 19. Department of Health and HumanServices, National Institutes of Health. NIH Pub. No. 07-6242. Bethesda, MD: USA: National Cancer Institute, 2008.

33. Wilson S. Research is ceremony: indigenous research methods. Halifax, NS, Canada: Fernwood, 2008

34. Canales MK, Geller BM. Moving in between mammography: screening decisions of American Indian women in Vermont. Qual Health Res 2004;14:836-57. 